Sophia Victoria Krebs, Wolfgang Lukas, Rüdiger Nutt-Kofoth, Anja Platz-Schliebs, Karl-Heinrich Schmidt, Uwe Stadler, Eric Steinhauer

\title{
Editions- und Dokumentwissenschaft an der Bergischen Universität Wuppertal
}

http://doi.org/10.1515/bd-2018-0104

Zusammenfassung: Der Masterstudiengang „Editions- und Dokumentwissenschaft" vermittelt philologische Kompetenzen in Theorie und Praxis der Edition, im Bereich der philologischen Textkritik wie auch in dem der Dokumentverarbeitung. Die wissenschaftlich gesicherte Herstellung, Erschließung und Repräsentation von Texten und ihrer kulturellen Überlieferung steht dabei im Fokus. Im Studiengang werden zudem u.a. modernes informations- und medientechnologisches Wissen, Medienrecht, Typographie und elektronisches Publizieren gelehrt.

Schlüsselwörter: Editionswissenschaft, Textkritik, Medienphilologie und -technologie

Abstract: The master programme 'Editorial Studies and Document Science' offers an in-depth training in philological skills required for the theory and practice of editing, philological criticism and document processing. The focus is on scientifically sound methods of producing, recovering and representing texts and their cultural heritage. The degree course also specializes in modern information and media technologies, media rights, typography and electronic publishing.

Keywords: Editorial studies, textual criticism, media philology and technology

\footnotetext{
Sophia Victoria Krebs: krebs@uni-wuppertal.de

Wolfgang Lukas: wlukas@uni-wuppertal.de

Rüdiger Nutt-Kofoth: nuttkofo@uni-wuppertal.de

Anja Platz-Schliebs: platz-schliebs@bib.uni-wuppertal.de

Karl-Heinrich Schmidt: karl-heinrich.schmidt@dmt.uni-wuppertal.de

Uwe Stadler: stadler@uni-wuppertal.de

Eric Steinhauer: direktion.ub@fernuni-hagen.de
} 


\section{Einleitung}

Zum Wintersemester 2010/11 wurde an der Bergischen Universität Wuppertal der Master-Studiengang „Editions- und Dokumentwissenschaft“ eingerichtet. Er hat sich seitdem als einer der erfolgreichsten Fach-Master der Fakultät für Geistes- und Kulturwissenschaften etabliert. Neben dem Studiengang „Editionswissenschaft und Textkritik“ an der Universität Heidelberg ist der sowohl interfakultär als auch interdisziplinär konzipierte Studiengang derzeit bundesweit der einzige dieser Art. Er kombiniert eine geistes- und kulturwissenschaftliche Grundausrichtung mit medientechnologischen Anteilen und bündelt die Vermittlung folgender vier Kompetenzfelder: 1. Editionsphilologie, 2. Informations- und Medientechnologie, 3. Mediengestaltung/-design, 4. Medien- (speziell Archiv-, Verlags-, Urheber- etc.) Recht. Damit profiliert sich die Wuppertaler Editions- und Dokumentwissenschaft als moderne, medien- und informationstechnologisch gestützte Kulturwissenschaft.

\section{Das Fach: Definition und Selbstverständnis}

Die wissenschaftliche Behandlung von Editionen wie die von Dokumenten erfolgt (auch nach dem Selbstverständnis der jeweiligen Communities) transdisziplinär und in theoretischer Perspektive. Für die editorische und dokumentenverarbeitende Praxis wird die Konstitution (,Herstellung'), Erschließung (z.B. Kommentierung) und Repräsentation von (oft kulturell hochbewerteten) Texten und Dokumenten zum Zwecke ihrer (Wieder-)Verfügbarmachung theoretisch aufgearbeitet. Speziell die Editionswissenschaft stellt daher für Texte die Basis aller Philologien und sonstiger auf Texten bzw. ,Volltextdokumenten' basierender Disziplinen, etwa der Philosophie, der Theologie, der Geschichtswissenschaft, dar; sie ist aber ebenso wesentlich für Disziplinen, die auf anderen Zeichensystemen bzw. Notationsformen beruhen wie z.B. die Musikwissenschaft, Bildwissenschaft, Filmwissenschaft etc. Speziell die Dokumententheorie stellt zusätzlich zu ihrer technischen Fundierung die Begriffsapparate für die jeweiligen Publikationsmöglichkeiten bereit.

Innerhalb der Philologien gilt die Editionswissenschaft als die erste und älteste Grundlagenforschung. Editionswissenschaftliche Operationen wurden schon in der Antike betrieben. Bei der Entstehung der Germanistik im frühen 19. Jahrhundert bildete die Erschließung von Texten und Quellen in Editionen den zunächst wichtigsten Bestandteil. Die Editionswissenschaft gilt heute als einer der drei „disziplinären Kerne der [literaturwissenschaftlichen] Germanistik“ 
neben der Literaturgeschichtsschreibung und der Interpretation und steht dabei nicht nur an der ersten Stelle dieser drei Grundpfeiler, sondern diese Zuordnung wird zugleich als „relativ unstrittig“ angesehen, sodass der Editionswissenschaft ein ,in legitimatorischer Hinsicht insgesamt vergleichsweise unangefochtene[r] Status“ zugeschrieben wird. ${ }^{1}$ Ihr transdisziplinärer Charakter zeigt sich aber auch darin, dass sie die Sprachgrenzen überschreitet und ebenso für die nichtdeutschsprachigen Philologien die Basisforschung in ihrer je fachspezifischen Ausprägung darstellt (Textual Scholarship/Scholarly Editing im anglo-amerikanischen Raum, critique génétique in Frankreich). Sie ist damit nicht nur interdisziplinär, sondern auch international orientiert.

Vermittelt die Philologie im angegebenen Sinne Fähigkeiten zur Erzeugung von fertigem ,content', hat die Dokumentverarbeitung dessen Modellierung und technische Gestaltung auf dieser Basis im Blick. Forschungen zur Theorie des Dokuments definieren die Einbettung von ,content‘ in Prozesse der Informationsverarbeitung. Dokumentverarbeitung unterscheidet sich von der reinen Datenverarbeitung dadurch, dass sie zumeist semiformatierte Daten (in natürlicher Sprache, Graphiken, Bewegtbild und Ton) für einen menschlichen Leser auf unterschiedlichsten Darstellungsmedien - vom Papyrus bis zum Bildschirm und elektronischen Datenträger - bereitstellt.

\section{Der Masterstudiengang - Konzept und Profil}

Der Wuppertaler Studiengang ist in eine einschlägige Forschungsumgebung eingebettet, die gebildet wird durch das Interdisziplinäre Zentrum für Editions- und Dokumentwissenschaft (IZED), ${ }^{2}$ das ein Forum für den (inter)nationalen wissenschaftlichen Austausch über Fach- und Fachbereichsgrenzen hinweg etabliert hat, ferner durch das seit 2016 laufende und in Kooperation mit der Kirchlichen Hochschule Wuppertal-Bethel durchgeführte DFG-Graduiertenkolleg zum Thema Dokument - Text - Edition. Bedingungen und Formen ihrer Transformation und Modellierung in transdisziplinärer Perspektive ${ }^{3}$ sowie durch zahlreiche laufende editorische Forschungsprojekte. ${ }^{4}$

1 Strohschneider, Peter; Vollhardt, Friedrich: Interpretation. Eine Einleitung in den Thementeil dieses Heftes. In: Mitteilungen des Deutschen Germanistenverbandes 49 (2002), H. 2: „Interpretation“, S. 98-102, hier: S. 100.

2 https://www.ized.uni-wuppertal.de/home.html [Zugriff: 03. 09. 2018].

3 https://www.editionen.uni-wuppertal.de [Zugriff: 03. 09. 2018].

4 https://www.ized.uni-wuppertal.de/home/editionsprojekte.html [Zugriff: 03. 09. 2018]. 
Die Transdisziplinarität des Studiengangs wird anhand der verschiedenen beteiligten Fächer deutlich: Beheimatet in der Germanistik bzw. Literaturwissenschaft und der Druck- und Medientechnologie, setzt sich der Studiengang zudem in verschiedener Gewichtung aus Veranstaltungen der Anglistik, der Geschichte, der Klassischen Philologie, der Philosophie, der Theologie, der Mediengestaltung, der Universitätsbibliothek sowie externen Experten ${ }^{5}$ aus dem Medienrecht, dem Archivwesen sowie dem Verlagswesen zusammen.

\subsection{Studium}

Nach (bzw. z. T. neben) dem Studium des philologischen Kernbereichs (vier Pflichtmodule) schließt sich ein Profilbereich (drei Wahlpflichtmodule) an, in dem die Möglichkeit der Schwerpunktsetzung für ein philologisches oder ein medientechnologisches Studienprofil besteht. Die vorgeschriebene Kombination - zwei Wahlpflichtmodule aus dem gewählten Schwerpunktbereich plus eines aus dem nicht gewählten Bereich - gewährleistet dabei die angestrebte Verzahnung von Philologie und Informationsverarbeitung, die in diesem Studiengang modellhaft realisiert wird. Adressaten dieses MA-Studiengangs sind alle Studierenden, die im BA mindestens eine ,Textwissenschaft‘ (etwa eine Philologie, Philosophie, Geschichte, Theologie) erfolgreich studiert haben.

Wenngleich dominant forschungsorientiert, weist der Wuppertaler Studiengang der Praxis einen hohen Stellenwert zu. Praktische und anwendungsbezogene Fertigkeiten in allen vier Kompetenzbereichen sind integraler Bestandteil des editions- und dokumentwissenschaftlichen Selbstverständnisses. Dem trägt der Studiengang zum einen durch spezifische Lehrveranstaltungen Rechnung, etwa durch (mindestens) ein umfangreiches Projektseminar, in dem die theoretischen Kenntnisse im Rahmen eines konkreten Editionsprojektes angewandt werden sollen, oder durch Übungen: von philologischen Transkriptionsübungen über Übungen zur Textauszeichnung mit XML/TEI und zur Satzherstellung bis hin zu praktischen Arbeiten in Editorial Design, um nur einige zu nennen (s. Curriculum). ${ }^{6}$ Zum anderen ist ein obligatorisches Praktikum von mindestens sechs Wochen zu absolvieren, dessen Ziel vor allem der Erwerb von konkreten Erfahrungen in außeruniversitären Betrieben bzw. Einrichtungen ist, die zugleich ein potentielles zukünftiges Berufsfeld darstellen. Die Lehrenden verfügen über

5 Aus praktischen Gründen wurde in Fällen, in denen keine geschlechtsneutralen Ersatzwörter gefunden werden konnten, das generische Maskulinum verwendet, wenngleich freilich stets sämtliche Geschlechter gemeint sind.

6 https://www.edw.uni-wuppertal.de/home/studium.html [Zugriff: 03. 09. 2018]. 
Kontakte zu Archiven, Bibliotheken, Medienunternehmen, die hierfür als Kooperationspartner zur Verfügung stehen (so u.a. um nur einige aus dem zentralen Bereich der Archive zu nennen, mit denen die Lehrenden im Rahmen von Editionsprojekten zusammenarbeiten bzw. gearbeitet haben: Deutsches Literaturarchiv Marbach; Heinrich-Heine-Institut Düsseldorf; Stadtarchiv Wuppertal; Friedrich-Engels-Haus/Historisches Zentrum Wuppertal; Goethe-Museum Düsseldorf; Landesarchiv NRW, Abt. Rheinland, Düsseldorf; Lippische Landesbibliothek Detmold; Goethe- und Schiller-Archiv Weimar; Staatsbibliothek Preußischer Kulturbesitz (Haus 2) Berlin; Bayerische Staatsbibliothek München; Schweizerisches Literaturarchiv Bern; Brüder-Grimm-Museum Kassel; Stadtarchive Kassel und Fulda; Hessisches Staatsarchiv Marburg; zudem Wirtschafts- und Firmenarchive). Weitere Praktikums- sowie potentielle Arbeitsstellen sind etwa Museen (wie das Filmmuseum Düsseldorf, das Von der Heydt-Museum Wuppertal oder das Beethovenhaus in Bonn) oder Verlage (wie der Emons Verlag, der DuMont Buchverlag oder der Wallstein Verlag).

Die Abschlussarbeit (Masterthesis) kann ihrerseits dominant theoretisch bzw. historisch-systematisch oder zuvorderst praktisch-editorisch oder aber auch empirisch orientiert sein. So sind etwa Untersuchungen zum Problembereich der Repräsentation von Materialität oder handschriftlicher Räumlichkeit in Editionen oder zu einzelnen Editionspositionen wie etwa den Registern oder dem Kommentar vorgelegt worden. Zudem sind vielfältige Editionen, vor allem zu literarischen Werken, Briefen oder historischen Dokumenten, erarbeitet, aber etwa auch Interessen und Verhaltensweisen von Nutzern historisch-kritischer Ausgaben erfragt und ausgewertet worden. ${ }^{7}$

\subsection{Zwischen Philologie und Dokument(theorie)}

Die philologischen Kenntnisse machen innerhalb des Studiengangs die Kernkompetenz aus, die zur methodisch reflektierten und wissenschaftlichen Standards verpflichteten Herausgabe von Texten befähigt. Dies schließt grundlegendes textwissenschaftliches und semiotisches Wissen ebenso ein wie Wissen über die historischen und insbesondere die mediengeschichtlichen Bedingungen von Überlieferung, Tradierung und Kanonisierung als kulturkonstitutiver Prozesse. Darauf baut als zentrale editionswissenschaftlich-philologische Kompetenz die Textkritik, d.h. die Fähigkeit zur ,kritischen‘ Konstitution und Herstellung von

7 Siehe die Auswahlliste auf https://www.edw.uni-wuppertal.de/home/studium/abschlussar beiten.html [Zugriff: 03. 09. 2018]. 
Texten, auf. Entsprechend neueren Entwicklungen in der editorischen Theorie und Praxis der letzten Jahrzehnte, die die Dimension der Textualität zunehmend um die der Materialität und Medialität als potentiell bedeutungskonstitutiver Komponenten des Textes erweitert haben, ${ }^{8}$ gilt auch im Kontext des material turn ein besonderes Augenmerk der Editorik im Spannungsfeld von ,Dokumentation“ und ,Interpretation'. Dies tangiert nicht nur die Felder der historischen Hilfswissenschaften wie etwa Paläographie, Kodikologie, Epigraphik, Diplomatik, die ebenfalls teilweise Bestandteil des Curriculums sind, sondern auch andere materielle Phänomene wie etwa Gebrauchsspuren, Auszeichnungen und Gestaltungsaspekte von Dokumenten. Im Studium werden die Sinne für den (medien-) historischen Kontext von Text und seinen Erscheinungsformen sensibilisiert, der ihrerseits Einfluss auf die Interpretation des graphemischen Textes haben.

Im Zuge des medientechnischen Wandels gewinnt die medien- und informationstechnologische Kompetenz eine wachsende Bedeutung. Sie soll zur methodisch reflektierten Herstellung von Editionen/Ausgaben mit unterschiedlichen materiellen Trägern von Dokumenten (Bildschirm, Papier, etc.) befähigen. Zentral sind insbesondere die Beherrschung normierter Plansprachen und Metasprachen zur digitalen Auszeichnung - u.a. XML als anwendungsneutrale Metasprache zur Beschreibung und Strukturierung von Dokumenten (als internationaler Standard vom W3C definiert und in Archiven, Bibliotheken, Editionen zunehmend eingesetzt) - und zur Satzherstellung sowie das Wissen über die digitale Medienproduktion im engeren Sinne (Druckvorstufe und Workflow-Management). Die Vermittlung dieser Einzelkompetenzen muss in einer universitären Ausbildung theoriegeleitet erfolgen. Dem dient die Behandlung forschungsnaher dokumententheoretischer Fragen, die sich gezielt an der Schnittstelle von Philologie und Informationsverarbeitung situieren und die von beiden Disziplinen vermittelten Kompetenzen - die philologische und die medientechnologische - systematisch aufeinander zu beziehen versuchen.

Ist die Editionsphilologie also auf den ,Inhalt‘ von Dokumenten ausgerichtet (im weitesten, Textualität und Materialität inkludierenden Sinn), so liefert die Dokumentenverarbeitung und ihre Theorie komplementär Beiträge zur Struktur von Dokumenten; Mediendesign/-technik schließlich steuert theoretisches und praktisches Wissen zur Gestaltung und Präsentation der ,Oberfläche“ von Dokumenten bei. Damit wird die Trias ,Logik - Layout - Inhalt‘ im Wuppertaler Stu-

8 Vgl. u. a.: Schubert, Martin (Hg.): Materialität in der Editionswissenschaft. Berlin 2010 (Beihefte zu editio, 32); Bohnenkamp-Renke, Anne (Hg.): Medienwandel/Medienwechsel in der Editionswissenschaft. Berlin 2012 (Beihefte zu editio, 35); Lukas, Wolfgang; Nutt-Kofoth, Rüdiger; Podewski, Madleen (Hg.): Text - Material - Medium. Zur Relevanz editorischer Dokumentationen für die literaturwissenschaftliche Interpretation. Berlin 2014 (Beihefte zu editio, 37). 
diengang vollständig abgebildet. Hinzu kommen schließlich noch elementare juristische Kenntnisse. Der Studiengang unterscheidet sich diesbezüglich auch von Studiengängen zu ,Digital Humanities‘ (DH), wenngleich er mit diesen einen Schnittbereich bildet.

Mit diesem Profil bietet die BUW deutschlandweit als eine der ganz wenigen Universitäten eine professionelle Ausbildung sowohl in den Geistes- und Kulturwissenschaften als auch in Medien/Informationstechnologie sowie - ein Erbe der ehemaligen Gesamthochschule - in Mediendesign an. Kompetenzen in Mediendesign/-technik sind für die professionelle Gestaltung und Präsentation der ,Oberfläche‘ von edierten Texten erforderlich und betreffen im Wesentlichen Fähigkeiten zur visuellen Strukturierung und zur medien- und ausgabenspezifischen (print, screen, handheld etc.) Aufbereitung von Texten mittels Typografie und Layout. Im Rahmen eines speziell auf die Bedürfnisse der Editions- und Dokumentwissenschaft zugeschnittenen Typografie-Moduls vermitteln Mediendesigner in Theorie und Praxis grundlegende Fähigkeiten in Layout und Typografie, die im Rahmen eigener Projekte bereits im Studium angewendet werden können. Studierende verfügen nach dem erfolgreichen Abschluss der medientechnologischen und -gestalterischen Module über die Fähigkeit, Strukturen digitaler sowie analoger Texte und Objekte sowie deren Aufbereitung und Herstellung zu verstehen, zu konzipieren, zu codieren und letztlich zu produzieren.

\subsection{Modul „Archiv, Recherche und Recht“}

Nachstehend sei das Pflichtmodul „Archiv, Recherche und Recht“ als im vorliegenden Kontext besonders interessantes etwas eingehender beschrieben. Es setzt sich aus zwei Veranstaltungen zusammen: dem Seminar „Recherchekompetenz für die Geistes- und Kulturwissenschaften“, das von den Studierenden im ersten Semester zu absolvieren ist, und dem im 2. Semester zu besuchenden Seminar „Medienrecht“. Das Lernziel dieses Moduls besteht darin, den Studierenden spezifische Recherchekompetenz, elementare Kenntnisse über das Archiv- und Bibliothekswesen sowie Wissen über die juristischen Rahmenbedingungen einer Publikation zu vermitteln.

\subsubsection{Recherchekompetenz für die Geistes- und Kulturwissenschaften}

Um den Studierenden einen möglichst effektiven Einblick in die Archiv- und Bibliotheksarbeit zu geben, ist das Seminar inhaltlich breit gefächert. Es ist als Ringveranstaltung konzipiert, die von einschlägigen Experten der Universitäts- 
bibliothek Wuppertal (Direktion und Fachreferenten), des Universitätsarchivs Wuppertal sowie des Archivs des Landschaftsverbands Rheinland (LVR) an den jeweiligen Wirkungsstätten abgehalten wird. Strukturell gliedert sich das Seminar in einen Archivteil und einen Bibliotheksteil, die vom Universitätsarchiv bzw. der Universitätsbibliothek organisiert werden.

\section{Der Archivteil}

In Hinsicht auf das Archivwesen gibt das Seminar einen Überblick über die Entstehung und Geschichte von Archiven von der Antike bis hin zu den Archivperspektiven zukünftigen ,E-Governments'. Es werden die Aufgaben und Strukturen von Archiven dargestellt, wie sie sich in aktuellen Archivgesetzen, -typen und -ordnungen niederschlagen, und die Arbeitsweisen von Archiven näher beleuchtet. Dabei werden die Grundlagen wie das Provenienzprinzip, die archivalische Bewertung, Tektonik, Bestandsbildung und Findbucherstellung erläutert.

Während einer eintägigen Exkursion ins Archiv des Landschaftsverbands Rheinland in Brauweiler werden die Studierenden in die Adelsarchivpflege eingeführt und führen praktische Übungen zur Erschließung an Archivalien durch. Es werden grundlegende Strategien sowie Probleme bei der Archivierung digitaler Unterlagen vermittelt; zudem wird das OAIS-Referenzmodell ${ }^{9}$ vorgestellt, der wichtigste Standard für die elektronische Archivierung. Schließlich werden die Studierenden am Beispiel einschlägiger Portale und Internetseiten in die digitale Archivierung eingeführt und befassen sich mit Digitalisaten und genuin digitalen Objekten. Im Rahmen von Workshops und Kurzreferaten werden die gewonnenen Erkenntnisse vertieft.

\section{Der Bibliotheksteil}

Im Bibliotheksteil werden zunächst die verschiedenen Typen von Bibliotheken, ihre Trägerschaften sowie Aufgaben und Ziele vorgestellt. Thematisiert werden Nationalbibliotheken, Landes-, Staats- und Regionalbibliotheken, Hochschulbibliotheken, öffentliche Bibliotheken und Spezialbibliotheken. Im Anschluss daran werden die wichtigsten Organisationen des Bibliothekswesens wie der Deutsche Bibliotheksverband (DBV) und seine Sektionen, die Bibliotheksverbünde oder der Verband der Bibliotheken des Landes NRW (VBNW) vorgestellt. Schließlich werden Evaluierungen, Prüfungen und Rankings im Bereich des Bibliothekswesens beleuchtet.

9 Das Akronym OAIS steht für Open Archival Information System. 
Eine grundlegende Voraussetzung für eine effektive und effiziente Literaturrecherche stellt die Kenntnis und strategische Beherrschung allgemeiner und fachlich relevanter Informationsmittel dar. Vor diesem Kontext widmet sich der Großteil des Bibliotheksteils ihrer Vermittlung. Einführend wird mit der Ermittlung von Publikationsarten sowie der Bewusstmachung von Recherchestrategien begonnen. Dies ist insbesondere essentiell für die Studierenden, die in ihrem Bachelor-Studium wenig systematisch und ausschließlich im Bibliothekskatalog ihrer Universität ihre Literatur gesucht haben. Es werden verschiedene Bibliothekskataloge mit und ohne kommerziellem Suchindex vorgestellt, spezielle Suchstrategien vermittelt und die Vorteile und Herausforderungen der Suche in Metakatalogen wie dem hbz-Verbundkatalog, dem Karlsruher Virtuellen Katalog (KVK) sowie dem WorldCat herausgearbeitet. Nach den Katalogen werden die Studierenden mit den wichtigsten allgemeinen, d.h., fächerübergreifenden gedruckten und elektronischen Bibliographien wie der Deutschen Nationalbibliographie (DNB), dem Verzeichnis der im deutschen Sprachraum erschienenen Drucke des 16./17. Jahrhunderts (VD 16/VD 17) und dem Gesamtverzeichnis des deutschsprachigen Schrifttums (GV neu/GV alt) vertraut gemacht.

Die folgenden Sitzungen befassen sich schließlich mit Fachinformationsmitteln und Fachinformationsdiensten. Es hat sich als besonders effektiv herausgestellt, die fachliche Auswahl nach dem Bachelor-Abschluss der Studierenden auszurichten, dessen Fokus im Bereich der Sprach- und Literaturwissenschaften, Geschichte und Pädagogik liegt. Geschult werden die Studierenden von den zuständigen Fachreferenten der Bibliothek, die auch verschiedene Übungsaufgaben mit ihnen durchführen. Im Bereich der Fachdatenbanken werden vor allem die MLA International Bibliography, die BDSL, die BLLDB sowie das digitale Bildarchiv für Kunst- und Kulturwissenschaften Prometheus behandelt. Das Phänomen Fachinformationsdienst (FID) wird an dem FID Erziehungswissenschaft und Bildungsforschung, FID Romanistik und FID Geschichtswissenschaft vorgestellt. Um eine optimale Literaturverwaltung zu gewährleisten, erhalten die Studierenden zudem eine Kurzeinführung in Citavi, dem Literaturverwaltungsprogramm, für das die Universitätsbibliothek Wuppertal eine Campuslizenz erworben hat.

Ein weiterer Schwerpunkt des Bibliotheksteils befasst sich mit den Aufgaben von Bibliotheken im Bereich elektronischer Publikationen. Nach einer Typologie der Publikationsmedien wird auf Publikationsplattformen eingegangen, die exemplarisch am Hochschulschriftenserver der Bergischen Universität Wuppertal näher dargelegt werden. Dabei stehen Hochschulpublikationen wie Dissertationen, Habilitationsschriften und Arbeitsberichte im Mittelpunkt der Darstellung. Exemplarisch lassen sich die „Schumpeter Discussion Papers“ anführen, eine wirtschaftswissenschaftliche Schriftenreihe, die seit 2008 ausschließlich 
online auf dem Hochschulschriftenserver erscheint. Vor diesem Kontext wird das Publikationsmodell „Open Access“ kontrovers diskutiert, einer Strategie, die auf nationaler und internationaler Ebene verfolgt wird, um den Wandel der wissenschaftlichen Publikationslandschaft aktiv zu gestalten.

Mit der Langzeitarchivierung wird ein weiterer prominenter Aspekt elektronischer Publikationen vermittelt. Die Studierenden werden mit den Chancen vertraut gemacht, die die Langzeitarchivierung mit sich bringt, aber auch mit den Herausforderungen, die insbesondere aus der Kurzlebigkeit vieler Datenträger, Formate, Software etc. resultieren. Schließlich wird mit dem Urheberrechtsgesetz und dessen Schranken eine Thematik behandelt, deren Kenntnis für die angehenden Dokument- und Editionswissenschaftler unerlässlich ist.

Den Abschluss des Seminars bildet eine Bibliotheksführung sowie eine Begehung des Rara-Magazins der Universitätsbibliothek Wuppertal, bei der die Studierenden - neben der Besichtigung der Altbestände - die Möglichkeit haben, den literarischen Nachlass des irischen Schriftstellers und Schauspielers Walter Macken (1915-1967) zu begutachten - nicht zuletzt auch im Hinblick auf zukünftige Editionsprojekte.

\subsubsection{Medienrechtliche Aspekte}

Zum Studiengang der Editions- und Dokumentwissenschaft gehören auch rechtliche Fragestellungen. Da die editorische Praxis stets mit fremden geistigen Schöpfungen zu tun hat, sind bei jüngeren Materialien solide Kenntnisse in den Grundlagen des Urheberrechts unerlässlich. Zudem schützt das Recht wissenschaftliche Editionen, die sich von bereits vorhandenen Ausgaben unterscheiden, mit einem eigenen Leistungsschutzrecht. Gleiches gilt bei der Erstpublikation bislang unveröffentlichten Materials, dessen Urheber vor mehr als 70 Jahre verstorben ist. Damit erweist sich bereits das Edieren als ein in mehrfacher Hinsicht rechtsrelevanter Vorgang. Weitere Berührungspunkte zum Recht ergeben sich aus den unterschiedlichen Benutzungsbestimmungen der Gedächtnisinstitutionen, wobei hier vor allem das Archivrecht besondere Aufmerksamkeit verdient, sowie aus der auch in der Editions- und Dokumentwissenschaft sehr präsenten Digitalisierung. Dabei sollten nicht nur Rechtsfragen der Datenbanken oder des Datenschutzrechts in Grundzügen bekannt sein. Auch neue Publikationsformen wie das Open-Access-Publizieren oder generell die Netzpublikation werden ohne ihre juristischen Implikationen nur unzureichend erfasst. Anders als körperliche Medien sind digitale Inhalte in ihrer nutzbaren Medialität ganz entscheidend durch ihre rechtliche Situation geprägt, die wiederum ohne urheberrechtliche Kenntnisse nicht verstanden werden kann. Weiterhin fordert der Aufstieg des 
Internet zum neuen Leitmedium der Gegenwart das kulturelle Gedächtnis in besonderer Weise heraus. Nicht nur die technische Haltbarkeit der Netzpublikation, auch ihre rechtliche Zugänglichkeit und ihre Authentizität werden zu einem Problem. Da die Editions- und Dokumentwissenschaft traditionellerweise an wichtigen Überlieferungsprozessen des kulturellen Gedächtnisses beteiligt ist, ist sie von diesen künftigen Entwicklungen betroffen und muss daher auch die rechtlichen Implikationen des digitalen kulturellen Gedächtnisses verstehen und nachvollziehen.

Rechtsfragen sind für die Editions- und Dokumentwissenschaft aber nicht nur ein instrumentelles Thema, das in der beruflichen Anwendung nützlich und in gewisser Weise auch unverzichtbar ist. Auch in überlieferungsgeschichtlicher Hinsicht sind Fragen der Zensur oder der Persönlichkeitsrechte wichtig, um eine bestimmte Werkgestalt oder besondere Publikationsstrategien erklären und verstehen zu können. In dieser Hinsicht kann die Rechtswissenschaft historisches Grundlagenwissen für die editorische Arbeit bereitstellen.

Der wohl spannendste Bereich des Verhältnisses von Editions- und Dokumentwissenschaft zur Jurisprudenz liegt aber in ihrem kritischen Potential eingeführten Rechtsbegriffen gegenüber. Wenn etwa das Urheberrecht mit Konzepten von ,Werk' oder ,Autorschaft“ operiert, so spiegeln sich darin bestimmte geistesgeschichtliche Situationen, die mit guten Gründen kritisiert und gerade angesichts der neuen Herausforderungen durch den digitalen Wandel auch infrage gestellt werden können. Hier vermag die Editions- und Dokumentwissenschaft eigene Theorieangebote $\mathrm{zu}$ machen und damit auch die rechtswissenschaftliche Diskussion zu bereichern.

Es gibt also viele Gründe, Rechtsfragen zum Gegenstand des editions- und dokumentwissenschaftlichen Studiums zu machen. Im Wuppertaler Studiengang geschieht dies in Form einer intensiven Einführungsvorlesung, bei der die Grundlagen des Urheberrechts, die Leistungsschutzrechte des Edierens sowie die Rechtsfragen des digitalen Wandels im Vordergrund stehen.

\section{Berufsfelder}

Der Theorie und Praxis integrierende Studiengang bietet gezielt und gebündelt eine neuartige Kombination von Kompetenzen mit hoher Anschlussfähigkeit und beruflicher Wertigkeit. Er vermittelt insbesondere Schlüsselqualifikationen für alle professionell Dokumente verarbeitenden Berufe, sowohl für Tätigkeiten im akademischen Bereich - etwa im Rahmen von Forschungs/Editionsprojekten als auch in Verlagen, Redaktionen und Medienunternehmen sowie, ggf. mit ent- 
sprechender Weiterqualifizierung, in Bibliotheken und Archiven. Berufsmöglichkeiten ergeben sich aber auch im Zuge der tiefgreifenden Umstrukturierung des Publikationswesens: Verlage definieren sich mittlerweile zunehmend über bloße Distributions- und Marketingfunktionen und delegieren ursprünglich genuine Verlagstätigkeiten an ein spezifisches Dienstleistungsgewerbe (Büros/Agenturen für Satz, Layout und die Druckvorstufenherstellung; Informatiker mit philologischen Kompetenzen bzw. Philologen mit Informatik-Kompetenzen - oftmals mit sehr speziellen individuellen Kenntnissen und Fertigkeiten). Erfahrungsgemäß werden Absolventen des Studiengangs nicht nur gerne in wissenschaftlicher bzw. forschender Umgebung, sondern auch auf dem privaten Sektor angestellt. Die im Studium gewonnenen Kenntnisse ermöglichen Tätigkeiten unter anderem bei Medienunternehmen wie etwa Publishing-Firmen, Mediadatenanalysten, Mediaproduktionsfirmen sowie in diversen Unternehmen in Bereichen der Archivierung, Record Retention, Recherche und Dokumentation.

Ein für die Absolventen der Editions- und Dokumentwissenschaft noch wenig erschlossenes, aber künftig sicher interessanter werdendes Betätigungsfeld stellen die wissenschaftlichen Bibliotheken dar. Traditionell führt der Weg $\mathrm{zu}$ einer Beschäftigung in wissenschaftlichen Bibliotheken entweder über ein Bibliotheksreferendariat oder ein meist berufsbegleitendes postgraduales Masterstudium. Das herkömmliche Tätigkeitsfeld ist danach in der Regel das Fachreferat, also die Betreuung des Bestandsaufbaus in einem bestimmten Fach, verbunden mit vielfältigen Beratungs- und Schulungsaufgaben rund um den eigenen Bestand. Als Folge des auch in den Bibliotheken massiv präsenten digitalen Wandels tritt die Bestandspflege im Fachreferat zugunsten anderer Aufgaben immer mehr in den Hintergrund.

Jetzt werden verstärkt Fragen des digitalen Publizierens oder des Forschungsdatenmanagements bearbeitet, verbunden mit einem starken Engagement im Bereich der Medien- und Informationskompetenz. Eine besondere Herausforderung für Bibliotheken stellen dabei die Digital Humanities dar. Waren die Geisteswissenschaften stets den Bibliotheken und ihren Beständen, vor allem aber den Büchern eng verbunden, so lockert sich diese Beziehung gerade. Zudem werden vermehrt digitale Textkorpora gewünscht, an denen mit quantitativen Methoden der Informatik Beobachtungen und Untersuchungen gemacht werden. Bibliotheken sehen sich in dieser Situation als Partner der Wissenschaft, haben aber in der eigenen beruflichen Tradition nur sehr begrenzte Kompetenzen anzubieten, die hier unterstützen können. Die wichtigste Fähigkeit dürfte wohl die bibliothekarische Erfahrung mit Metadaten und Regelwerken der Verzeichnung sein. Daneben sind noch die mittlerweile recht ausgebauten digitalen Publikationsinfrastrukturen zu nennen, aus denen sich eine Expertise in elektronischer Langzeitarchivierung ableitet. 
Gleichwohl finden sich technische Arbeitsumgebungen für die Digital Humanities immer öfter in eigenen Einrichtungen. Manchmal sind diese „Digital Humanities Centers“ aber auch nur eine Abteilung einer wissenschaftlichen Bibliothek. Unabhängig von der institutionellen Ausprägung gibt es stets eine enge Kooperation zu den Bibliotheken, die ja das Material für die aller Computerphilologie vorgängige Digitalisierung bereitstellen, teilweise aber auch die Digitalisierung selbst durchführen. An dieser Schnittstelle zwischen Digitalisierung und Digital Humanities liegt ein interessanter Tätigkeitsbereich für Editions- und Dokumentwissenschaftler. Sie können Digitalisate strukturieren und die Erstellung der Forschungskorpora übernehmen. Ihre Kenntnisse in Überlieferungsprozessen und traditionellen Editionstechniken qualifizieren sie überdies für die Arbeit in Forschungsbibliotheken mit Nachlässen oder unikalem Sonderbestand.

In der wissenschaftlichen Bibliothek des digitalen Wandels können sie damit zum einen die Überlieferungsbildung und Zugänglichkeit des vorhandenen Bestandes auch in digitalen Kontexten gewährleisten und zum anderen diesen Bestand für digitale Forschungstechniken aufbereiten. Sie können damit für Digitalia und Sonderbestände gleichermaßen die Vermittlungsrolle zwischen Bibliothek und Wissenschaft übernehmen, die der traditionelle Fachreferent in der Buchwissenschaft alter Prägung innehatte, der publizierte Forschungsquellen für die wissenschaftliche Arbeit sammelte, erschloss und für die Nutzung zur Verfügung stellte.

Der Beruf des wissenschaftlichen Bibliothekars, der sich an den Master in Dokument- und Editionswissenschaft anschließen könnte, kann auf verschiedenen Wegen erreicht werden, die je nach Bundesland variieren. ${ }^{10}$ Neben der Ausbildung im Referendariat, d.h. der beamtenrechtlichen Ausbildung im Vorbereitungsdienst als Beamter auf Widerruf, und dem Volontariat besteht an einigen deutschen Hochschulen - wie beispielsweise der TH Köln - die Möglichkeit eines postgradualen Studiums der „Bibliotheks- und/oder Informationswissenschaft" mit dem Abschluss Master of Library and Information Science (MA LIS). Aufgrund der gegebenen formalen Regularien und Einstellungsvoraussetzungen für wissenschaftlich Beschäftigte ist es schwierig, eine pauschale Bewertung für die mögliche Verwertbarkeit des editions- und dokumentwissenschaftlichen Abschlusses im bibliothekarischen Berufsalltag vorzunehmen.

Der berufliche Einstieg für Editions- und Dokumentwissenschaftler in das Bibliothekswesen wird zunächst allein über Projektstellen erfolgen. In dem Maße jedoch, in dem sich neue digitale Forschungsservices als Regelaufgaben in Bib-

10 Nähere Informationen hierzu sind verfügbar auf der Website der Kommission für berufliche Qualifikation des VDB unter https://www.vdb-online.org/kommissionen/qualifikation/ausbildungsinfo/ [Zugriff: 03. 09. 2018]. 
liotheken, Hochschulen oder Forschungseinrichtungen etablieren, wird es auch Dauerstellen beim Stammpersonal für diese Fragestellungen geben, die nicht mehr mit traditionell ausgebildeten Bibliothekaren besetzt werden. Sofern eine hohe Affinität zu digitalen Themen vorhanden ist, sind Editions- und Dokumentwissenschaftler für die mit diesen Stellen verbundenen Aufgaben gut qualifiziert.

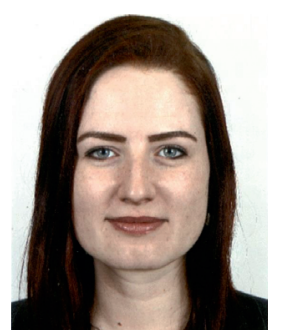

\section{Sophia Victoria Krebs M. A.}

Zentrum für Graduiertenstudien

Bergische Universität Wuppertal

Gaustr. 20

42119 Wuppertal

Deutschland

Mail: krebs@uni-wuppertal.de

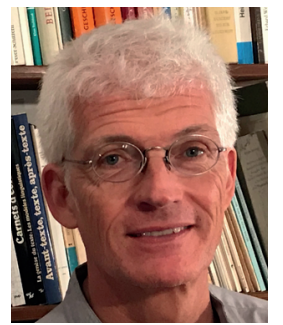

Prof. Dr. Wolfgang Lukas

Germanistik/Neuere deutsche Literaturgeschichte

Bergische Universität Wuppertal

Gaustr. 20

42119 Wuppertal

Deutschland

Mail:wlukas@uni-wuppertal.de

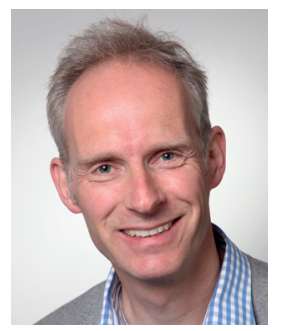

\section{Dr. Rüdiger Nutt-Kofoth}

Germanistik/Neuere deutsche Literaturgeschichte

Bergische Universität Wuppertal

Gaustr. 20

42119 Wuppertal

Deutschland

Mail: nuttkofo@uni-wuppertal.de

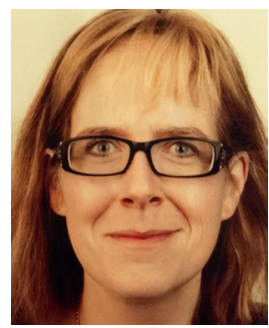

\section{Dr. Anja Platz-Schliebs}

Universitätsbibliothek

Bergische Universität Wuppertal

Gaustr. 20

42119 Wuppertal

Deutschland

Mail: platz-schliebs@bib.uni-wuppertal.de 


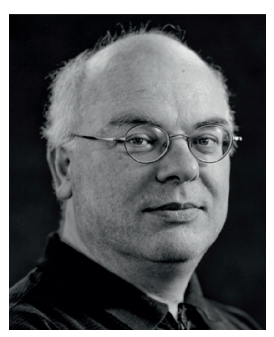

Prof. Dr. Karl-Heinrich Schmidt

Druck- und Medientechnologie

Bergische Universität Wuppertal

Campus Freudenberg

Rainer-Gruenter-Str. 21

42119 Wuppertal

Deutschland

Mail: karl-heinrich.schmidt@dmt.uni-wuppertal.de

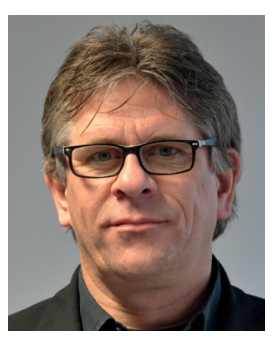

Uwe Stadler

Direktor der Universitätsbibliothek

Bergische Universität Wuppertal

Gaustr. 20

42119 Wuppertal

Deutschland

Mail: stadler@uni-wuppertal.de

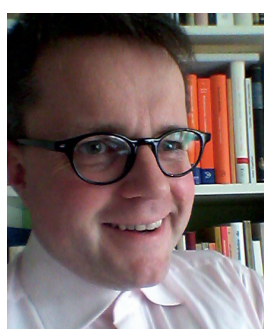

\section{Prof. Dr. Eric W. Steinhauer}

Stellvertretender Bibliotheksdirektor

Universitätsbibliothek der FernUniversität

Universitätsstr. 23

Gebäude 6

58097 Hagen

Deutschland

Mail: eric.steinhauer@fernuni-hagen.de 